Short Communication

\title{
Copper Toxicity Associated With an ATP7A-Related Complex Phenotype
}

\author{
Daniel Natera-de Benito, MD, PhD ${ }^{\mathrm{a}}$, Abel Sola, MSc ${ }^{\mathrm{b}}$, Paulo Rego Sousa, MD ${ }^{\mathrm{a}, \mathrm{c}}$, \\ Susana Boronat, MD, PhD ${ }^{\mathrm{d}}$, Jessica Expósito-Escudero, MD a , Laura Carrera-García, MD a, \\ Carlos Ortez, MD ${ }^{\mathrm{a}}$, Cristina Jou, MD ${ }^{\mathrm{a}, \mathrm{e}, \mathrm{f}}$, Jordi Muchart, MD ${ }^{\mathrm{g}}$, Monica Rebollo, MD ${ }^{\mathrm{g}}$, \\ Judith Armstrong, PhD a, f, h, Jaume Colomer, MD, PhD a, \\ Àngels Garcia-Cazorla, MD, PhD ${ }^{\mathrm{f}, \mathrm{i}}$, Janet Hoenicka, $\mathrm{PhD}{ }^{\mathrm{b}, \mathrm{f}}$, \\ Francesc Palau, MD, PhD ${ }^{\mathrm{b}, \mathrm{f}, \mathrm{h}, \mathrm{j},{ }^{*}, \dagger}$, Andres Nascimento, MD ${ }^{\mathrm{a}, \mathrm{f}, \dagger}$
}

\footnotetext{
${ }^{a}$ Neuromuscular Unit, Department of Pediatric Neurology, Hospital Sant Joan de Déu and Institut de Recerca Sant Joan de Déu, Barcelona, Spain

${ }^{\mathrm{b}}$ Laboratory of Neurogenetics and Molecular Medicine - IPER, Institut de Recerca Sant Joan de Déu, Barcelona, Spain

${ }^{\mathrm{c}}$ Pediatric Neurology Unit, Department of Pediatrics, Hospital Central do Funchal, Funchal, Portugal

${ }^{\mathrm{d}}$ Department of Pediatrics, Hospital Santa Creu i Sant Pau, Barcelona, Spain

e Department of Pathology, Hospital Sant Joan de Déu, Barcelona, Spain

${ }^{\mathrm{f}}$ Center for Biomedical Research Network on Rare Diseases (CIBERER), ISCIII, Madrid, Spain

${ }^{g}$ Department of Radiology, Hospital Sant Joan de Déu, Barcelona, Spain

${ }^{\mathrm{h}}$ Department of Genetic and Molecular Medicine - IPER, Hospital Sant Joan de Déu, Barcelona, Spain

${ }^{i}$ Department of Pediatric Neurology, Hospital Sant Joan de Deu, Barcelona, Barcelona, Spain

${ }^{j}$ Clinic Institute of Medicine \& Dermatology, Hospital Clínic, and Division of Pediatrics, University of Barcelona School of Medicine and Health Sciences, Barcelona, Spain
}

\section{A R T I C L E I N F O}

\section{Article history:}

Received 9 November 2020

Accepted 17 March 2021

Available online 26 March 2021

\section{Keywords:}

ATP7A

ATP7B

Copper replacement therapy

Distal hereditary motor neuropathy

Occipital horn syndrome

\begin{abstract}
A B S T R A C T
Background: The ATP7A gene encodes a copper transporter whose mutations cause Menkes disease, occipital horn syndrome (OHS), and, less frequently, ATP7A-related distal hereditary motor neuropathy (dHMN). Here we describe a family with OHS caused by a novel mutation in the ATP7A gene, including a patient with a comorbid dHMN that worsened markedly after being treated with copper histidinate. Methods: We studied in detail the clinical features of the patients and performed a genomic analysis by using TruSight One Expanded Sequencing Panel. Subsequently, we determined the ATP7A and ATP7B expression levels, mitochondrial membrane potential, and redox balance in cultured fibroblasts of Patient 1. Results: We found a novel ATP7A late truncated mutation p.Lys1412AsnfsX15 in the two affected members of this family. The co-occurrence of OHS and dHMN in Patient 1 reveals the variable phenotypic expressivity of the variant. A severe clinical and neurophysiologic worsening was observed in the dHMN of Patient 1 when he was treated with copper replacement therapy, with a subsequent fast recovery after the copper histidinate was withdrawn. Functional studies revealed that the patient had low levels of both ATP7A and ATP7B, the other copper transporter, and high levels of superoxide ion in the mitochondria.
\end{abstract}

Funding: This research was supported by Fundación Ramón Areces (grant no. CIVP18A3913), Fundación Amigos de Nono, Instituto Salud Carlos III Grant DTS16/00196, the

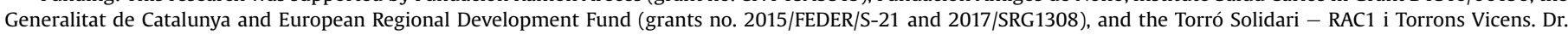

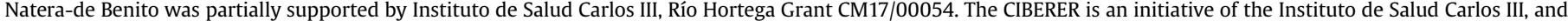
the Department of Genetic Medicine of Sant Joan de Déu Children's Hospital is part of the "Centre Daniel Bravo de Diagnòstic i Recerca de Malalties Minoritàries."

Conflicts of interest: None of the authors have any conflict of interest to disclose.

Ethics/consent to participation: This study was carried out following the guidelines of the Clinical Ethics Committee of Hospital Sant Joan de Déu (PIC 223-19). All participation was by signed informed consent.

Data availability: Any data not published within the article will be shared, upon reasonable request.

* Communications should be addressed to: Dr. Palau; Department of Genetic and Molecular Medicine; Hospital Sant Joan de Déu; Passeig Sant Joan de Déu, 2, Esplugues de Llobregat; 08950, Barcelona, Spain.

E-mail address: fpalau@sjdhospitalbarcelona.org (F. Palau).

Senior authors, equally contributed. 
Conclusions: Our findings broaden the clinical spectrum of ATP7A-related disorders and demonstrate that two clinical phenotypes can occur in the same patient. The copper-induced toxicity and low levels of both ATP7A and ATP7B in our patient suggest that copper accumulation in motor neurons is the pathogenic mechanism in ATP7A-related dHMN.

(C) 2021 Elsevier Inc. All rights reserved.

\section{Introduction}

ATP7A is a transmembrane protein that (1) exports copper across the plasma membrane to maintain intracellular copper homeostasis and (2) provides copper to copper-dependent enzymes in the trans-Golgi network. ${ }^{1}$ Mutations in the ATP7A gene may lead to three distinct $\mathrm{X}$-linked recessive clinical phenotypes: Menkes disease (MD, MIM \#309400), occipital horn syndrome (OHS, MIM \#304150), and ATP7A-related distal hereditary motor neuropathy (dHMN, MIM \#300489). The dHMN phenotype is a pure motor neuropathy in which, unlike MD and OHS, the copper-related biochemical parameters are normal. ${ }^{2,3}$ Although copper is relevant to maintain the integrity of motor neurons (MNs), the precise mechanisms by which dysfunctional ATP7A leads to the specific length-dependent axonal degeneration observed in MNs of patients with ATP7A-related dHMN remain unknown.

Here we present a family with OHS due to a novel ATP7A late truncating variant. In addition to OHS, the older patient has a dHMN that severely worsened while treated with copper replacement therapy, which may be key to understanding the pathogenic mechanism in ATP7A-related dHMN.

\section{Patient Descriptions}

Patient 1 is a 24-year-old male, the maternal uncle of Patient 2. He had experienced gait difficulties since age three years along with learning difficulties and symptoms of dysautonomia (syncope related to orthostatic hypotension and temperature instability). Physical examination at age 10 years revealed a high forehead, large ears, pectus excavatum, and normal hair (Fig 1A). Trichoscopy was not performed. His skin was lax and hyperextensible, and his joints were hypermobile. Distal muscle weakness in his lower limbs was detected (tibialis anterior: Medical Research Coucil scale grade 4-/ 5). Serum copper levels were $313 \mu \mathrm{g} / \mathrm{L}$ (normal range: 620 to 1544 $\mu \mathrm{g} / \mathrm{L}$ ) and ceruloplasmin level was $103 \mathrm{mg} / \mathrm{L}$ (normal range: 170 to $460 \mathrm{mg} / \mathrm{L}$ ). Occipital exostoses at the tendinous insertions of the sternocleidomastoid and trapezius muscles were observed in skull radiographs (Fig 1F). The brain angio-magnetic resonance imaging showed that anterior and middle cerebral arteries were elongated and tortuous (Fig 1B). The first electroneurography test (ENG), performed when the patient was aged 10 years, showed reduced amplitude of the compound motor action potentials (CMAPs) with normal velocity, whereas sensory nerve conductions were normal. Positive waves and fibrillations were observed via electromyography (EMG). All these findings confirmed an axonal neuropathy predominantly affecting motor nerves (Table). A frameshift variant ATP7A c.4236delA; p.Lys1412AsnfsX15 that causes a truncated peptide that preserves all ATP7A domains except the carboxyl terminal (Fig 1G) was detected after genomic DNA was analyzed using the TruSight One Expanded Sequencing Panel in a NextSeq 500 platform (Illumina, San Diego, California, USA). The unaffected mother was heterozygous for the variant. No pathogenic variants were identified in any of the other known causative genes for inherited neuropathies.
Patient 2 is a 10-year-old boy, son of Patient 1's sister. Examination at age 10 months showed global hypotonia, down-slanting eyes, internal epicanthus, prominent large ears, hypermobile joints, lax skin, and hair that was hypopigmented, dull, and friable (Fig 1D). Pili torti was observed through optic microscopy (Fig 1C). Mild motor delay was detected. Serum copper and ceruloplasmin were in the normal range ( $1123 \mu \mathrm{g} / \mathrm{L}$ [normal range: 620 to 1544 $\mu \mathrm{g} / \mathrm{L}$ ] and $225 \mathrm{mg} / \mathrm{L}$ [normal range: 220 to $470 \mathrm{mg} / \mathrm{L}$ ], respectively). Vascular tortuosity and dilatation of the Willis polygon and basilar and vertebral arteries were observed in a cerebral magnetic resonance imaging (Fig 1E). His ENG/EMG evaluation was normal, with peroneal CMAP and nerve conduction velocity within the normal range ( $2.6 \mathrm{mV}$ and $55.4 \mathrm{~m} / \mathrm{s}$, respectively). It is not known if Patient 2 had occipital exostoses because a skull x-ray was not performed.

Because some patients with MD had proven to be responsive to early copper replacement therapy ${ }^{4,5}$ and since copper supplementation may be potentially beneficial in OHS, ${ }^{6}$ we treated Patient 1 with copper histidinate at age 15 years, after having obtained an informed consent from the family. Doses of $20 \mu \mathrm{g} / \mathrm{kg}$ were administered three times per week and increased to four times per week after serum levels of copper and ceruloplasmin were tested. The treatment was withdrawn after four months due to a marked worsening of the motor symptoms, with increased distal muscular weakness in all four limbs. His gait worsened considerably, and significant difficulties to perform daily activities appeared. Actions such as pulling out a book from his bag, writing, or picking up a glass of water became very difficult for him, if not impossible. The ENG/EMG evaluation was repeated after four months of treatment, and it demonstrated a lower CMAP and clear signs of denervation (Table). Once the copper histidinate treatment was withdrawn, the adverse side effects disappeared progressively and rapidly until the functional status of the patient was completely recovered. Four months after the copper was withdrawn, the neurophysiologic evaluation showed similar results to those obtained in the ENG/EMG performed before the start of treatment (Table).

\section{Functional studies}

Because ATP7A and ATP7B are two copper transporters that act in a coordinated manner in neurons, ${ }^{7}$ we quantified their levels in fibroblasts of Patient 1 and compared them with the levels in fibroblasts of two patients with the main inborn errors of copper metabolism: MD and Wilson disease (WD), as well as the levels in fibroblasts of control individuals (Fig 2A and B). ATP7A showed small size and was poorly expressed in Patient 1 fibroblasts, absent in MD fibroblasts, and normal in controls and WD fibroblasts. Intriguingly, ATP7B was also significantly lower in Patient 1 than in the other individuals. Exome re-analysis of the ATP7B gene did not detect any pathogenic variants in Patient 1 , which suggests that this lower level of ATP7B could be related to the low expression of ATP7A ${ }^{\text {Lys1412AsnfsX15. }}$

As ATP7A is essential for redox balance in mitochondria, we quantified the levels of mitochondria superoxide (MitoSOX Red 


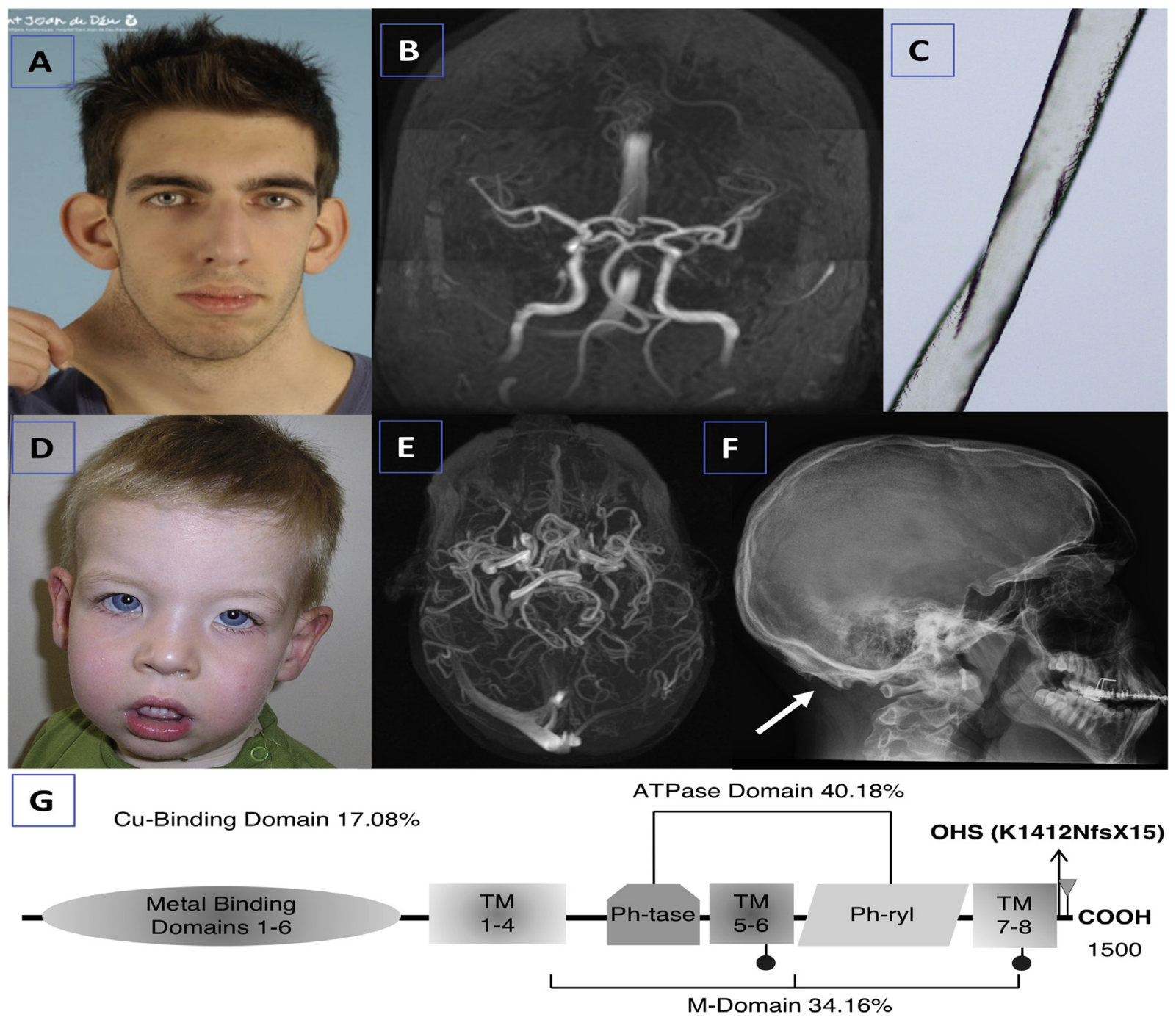

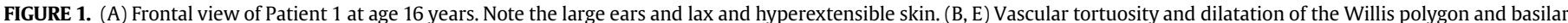

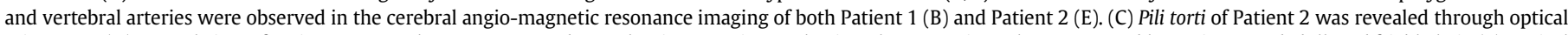

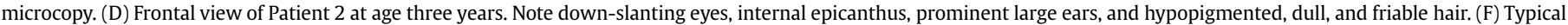

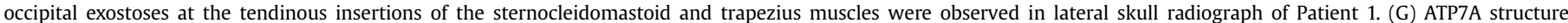

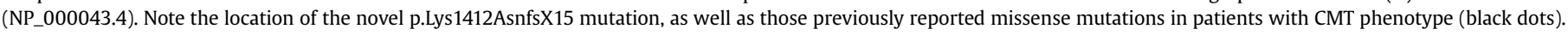
TM, transmembrane domains; Ph-tase, phosphatase domain; Ph-ryl, phosphorylation domain. The color version of this figure is available in the online edition.

reagent, Molecular Probes) and membrane potential (tetramethylrhodamine methyl ester perchlorate, Sigma-Aldrich) in patients' fibroblasts using flow cytometry. We observed high levels of superoxide ion in the mitochondria of all patients' fibroblasts (Fig 2C). In contrast, membrane potential was only affected in MD fibroblasts (Fig 2D). Further characterization of fibroblasts' redox status using glutathione quantification revealed normal levels of both reduced glutathione (GSH) and oxidized glutathione (GSSG) (GSH/GSSG-Glo Assay, Promega) in fibroblasts of Patient 1 and the patient with WD, whereas the patient with MD showed significantly higher levels of GSSG (Fig 2E and F). Collectively, these data showed important differences in both the correlation of ATP7A/ ATP7B expression and in oxidative status across patients with OHS, $\mathrm{MD}$, and WD.

\section{Discussion}

Here we describe two related patients with OHS, one of them with comorbid dHMN. Both patients carry the frameshift mutation
p.Lys1412AsnfsX15 in ATP7A, which affects the last intracytoplasmic ATP7A domain. Unlike other truncating ATP7A variants, which cause MD, late truncating variants located at the $\mathrm{C}$ - $\mathrm{t}$ lead to an OHS phenotype by removing the distal di-leucine motif $\mathrm{L}^{1477} \mathrm{~L}^{1478}{ }^{8}$ The lack of this motif, which is an endocytic signal for ATP7A recycling between the trans-Golgi network and the plasma membrane, leads to a truncated ATP7A predominantly located at the plasma membrane. ${ }^{9}$ The resulting ATP7A $A^{\text {p.Lys 1412AsnfsX15 }}$ shortage at the trans-Golgi network would limit the copper supply to the copper-dependent enzymes lysyl oxidase and dopamineß-hydroxylase, eventually leading to the typical signs of OHS. The finding of pili torti in Patient 2, with normal serum copper and ceruloplasmin, would also be explained by the intracellular mislocation of ATP7A. Pili torti structure is caused by malformation of keratin due to lack of sulfhydryl oxidase. ${ }^{10}$ Sulfhydryl oxidase typically functions in the endoplasmic reticulum, catalyzed by copper, to promote disulfide bond-forming reactions, which are necessary for keratin cross-linking. The prevalence of pili torti in patients with OHS is still unknown. On the other hand, normal 
TABLE.

Patient 1 Neurophysiologic Examinations

\begin{tabular}{|c|c|c|c|}
\hline $\begin{array}{l}\text { Biochemical and Neurophysiological } \\
\text { Parameters }\end{array}$ & $\begin{array}{l}\text { Before Cu Therapy (Basal } \\
\text { Values) }\end{array}$ & $\begin{array}{l}\text { During Cu Therapy ( } 4 \text { Months on } \\
\text { Treatment) }\end{array}$ & $\begin{array}{l}\text { After Cu Therapy ( } 4 \text { Months Without } \\
\text { Treatment) }\end{array}$ \\
\hline \multicolumn{4}{|l|}{ Copper serum levels $(\mu \mathrm{g} / \mathrm{L})$} \\
\hline Normal range $(620-1544 \mu \mathrm{g} / \mathrm{L})$ & 313 & 604 & 359 \\
\hline \multicolumn{4}{|l|}{ Nerve conduction study } \\
\hline $\mathrm{R}$ peroneal nerve CMAP $(\mathrm{mV})$ & 0.6 & 0.3 & 0.6 \\
\hline R peroneal NCV $(\mathrm{m} / \mathrm{s})$ & 47.3 & 35.8 & 34.5 \\
\hline L suralis SNAP $(\mu \mathrm{V})$ & 9.1 & 6.2 & 2.2 \\
\hline L suralis sensory NCV (m/s) & 56.7 & 48.2 & 62.5 \\
\hline \multicolumn{4}{|l|}{ EMG study } \\
\hline Fibrillations & $2 / 10$ & $2 / 10$ & NP \\
\hline Positive waves & $2 / 10$ & $2 / 10$ & NP \\
\hline Amplitude & ++ & ++ & $\mathrm{NP}$ \\
\hline Duration & ++ & +++ & NP \\
\hline Firing & - & - & NP \\
\hline
\end{tabular}

Abbreviations:

$\mathrm{CMAP}=$ Compound motor action potential

$\mathrm{Cu}=$ Copper

$\mathrm{L}=$ Left

$\mathrm{NCV}=$ Nerve conduction velocity

$\mathrm{NP}=$ Not performed

$\mathrm{R}=$ Right

SNAP $=$ Sensory nerve action potential
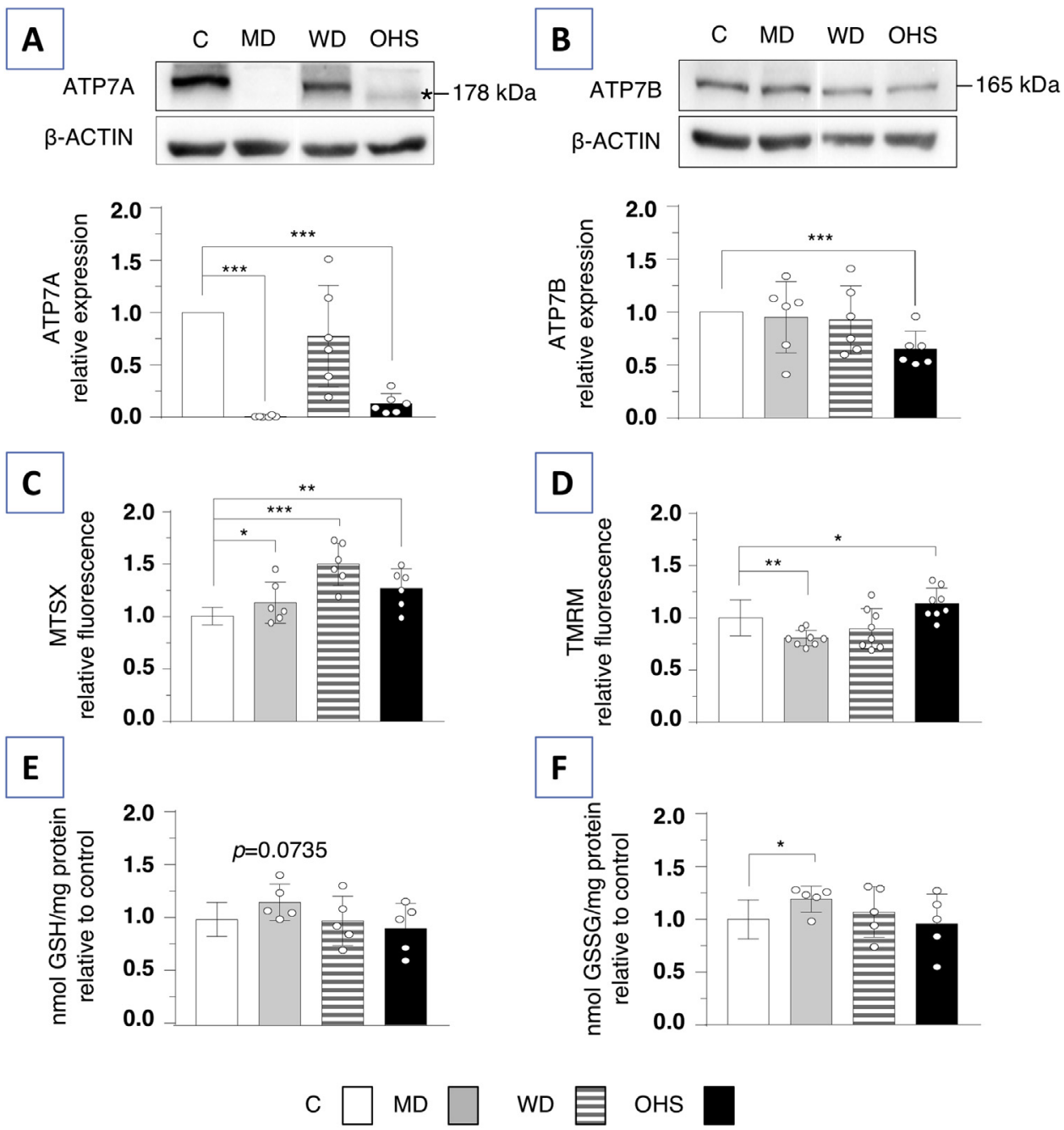

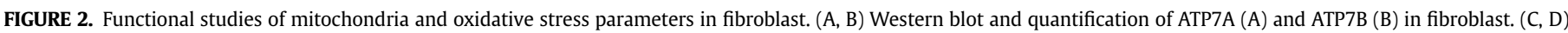

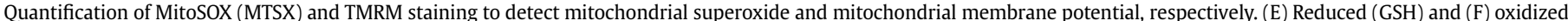

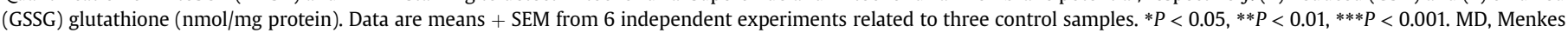

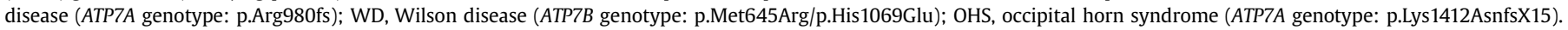


serum values of copper and ceruloplasmin have been reported in $29 \%$ and $26 \%$ of patients with OHS, respectively. ${ }^{11}$ This variability could be due to differences in the reduced amount of ATP7A variants and in their location, which could prevent the reduction of these biomarkers in some patients, in contrast to what is observed in the total absence of ATP7A in Menkes disease. In our family, the differences in copper management between Patients 1 and 2 , although they are uncles and nephews, may be related to individual genomic background.

The three missense variants in transmembrane domains that have been previously documented in patients with ATP7A-related dHMN also results in a preferential location of ATP7A at the plasma membrane. ${ }^{12}$ Therefore the accumulation of ATP7A at the plasma membrane seems a common pathophysiologic mechanism to explain the unique association of OHS and dHMN observed in Patient 1 . Moreover, one of the previously reported

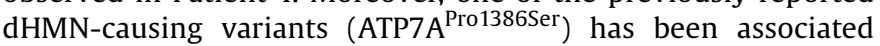
with reduced calcium $\left(\mathrm{Ca}^{2+}\right)$ removal and synapses recovery in NSC-34 cells. ${ }^{13}$ Since mitochondria contribute to $\mathrm{Ca}^{2+}$ buffering and energy production at synapses, ${ }^{14}$ it could be hypothesized that ATP7A-related dHMN variants impair mitochondrial transport, similar to what occurs in MFN2-related Charcot-MarieTooth disease. ${ }^{15}$ Putative mislocation of ATP7A at the plasma membrane would cause a decrease in the number of mitochondria at synapses, leading to synaptic dysfunction and neuropathy. Our comparison of the mitochondrial and cellular oxidative state in patients with MD and OHS supports the idea that impaired mitochondrial mobility, and not oxidative stress, would be the cause of dHMN in Patient 1.

Last, the finding of marked worsening of neuropathy in Patient 1 after copper supplementation could be also explained by the impaired trafficking of ATP7A, which would be unable to properly remove the excess of copper ${ }^{16,17}$ and would eventually lead to a copper accumulation in MNs and axons, which is a known pathogenic mechanism in neurodegenerative disorders. Patient 1 , who carries the p.Lys1412AsnfsX15 variant in ATP7A and has low levels of the other copper transporter ATP7B, would have a copper homeostasis defect in MNs that would have eventually resulted in both dHMN and toxicity after copper supplementation. In neurons, ATP7B is found in vesicles distributed throughout the cytosol where it sequesters copper and regulates the amount of cytosolic copper available for ATP7A. ${ }^{7}$ Further studies of the ATP7A/ATP7B ratio in additional patients' samples are necessary to better understand the biology of the functional relationship of these copper transporters. In any case, we propose that Patient 1 has a global defect in the regulation of intracellular copper levels that causes this particular OHS phenotype and eventually leads to axonal neuropathy that is highly susceptible to copper-induced toxicity. In contrast, we found in MD a total absence of ATP7A and normal levels of ATP7B. These differences in ATP7A/ATP7B ratio in MD and OHS could explain why copper replacement therapy may be beneficial in patients with MD and be toxic in patients with OHS. Therefore copper replacement therapy should be considered and monitored with caution in patients with dHMN and/or OHS according to the distinct pathophysiologic mechanisms discussed here.

\section{References}

1. Gourdon P, Liu XY, Skjorringe T, et al. Crystal structure of a copper-transporting PIB-type ATPase. Nature. 2011;475:59-64.

2. Gualandi F, Sette E, Fortunato F, et al. Report of a novel ATP7A mutation causing distal motor neuropathy. Neuromuscul Disord. 2019;29:776-785.

3. Kennerson ML, Nicholson GA, Kaler SG, et al. Missense mutations in the copper transporter gene ATP7A cause X-linked distal hereditary motor neuropathy. Am J Hum Genet. 2010;86:343-352.

4. Tang J, Donsante A, Desai V, Patronas N, Kaler SG. Clinical outcomes in Menkes disease patients with a copper-responsive ATP7A mutation, G727R. Mol Genet Metab. 2008;95:174-181.

5. Kim BE, Smith K, Petris MJ. A copper treatable Menkes disease mutation associated with defective trafficking of a functional Menkes copper ATPase. J Med Genet. 2003;40:290-295.

6. Kaler SG. ATP7A-related copper transport diseasesg-emerging concepts and future trends. Nat Rev Neurol. 2011;7:15-29.

7. Schmidt K, Ralle M, Schaffer T, et al. ATP7A and ATP7B copper transporters have distinct functions in the regulation of neuronal dopamine-hydroxylase. J Biol Chem. 2018;293:20085-20098.

8. Bonati MT, Verde F, Hladnik U, et al. A novel nonsense ATP7A pathogenic variant in a family exhibiting a variable occipital horn syndrome phenotype. Mol Genet Metab Rep. 2017;13:14-17.

9. Dagenais SL, Adam AN, Innis JW, Glover TW. A novel frameshift mutation in exon 23 of ATP7a (MNK) results in occipital horn syndrome and not in Menkes disease. Am J Hum Genet. 2001;69:420-427.

10. Fass D. The Erv family of sulfhydryl oxidases. Biochim Biophys Acta. 2008; 1783:557-566.

11. Beyens A, Van Meensel K, Pottie L, et al. Defining the clinical, molecular and ultrastructural characteristics in occipital horn syndrome: two new cases and review of the literature. Genes (Basel). 2019;10:528.

12. Yi L, Kaler S. ATP7A trafficking and mechanisms underlying the distal motor neuropathy induced by mutations in ATP7A. Ann N Y Acad Sci. 2014;1314: 49-54.

13. Yi L, Kaler SG. Direct interactions of adaptor protein complexes 1 and 2 with the copper transporter ATP7A mediate its anterograde and retrograde trafficking. Hum Mol Genet. 2015;24:2411-2425.

14. David G, Barett EF. Mitochondrial Ca2 + uptake prevents desynchronization of quantal release and minimizes depletion during repetitive stimulation of mouse motor nerve terminals. J Physiol. 2003;548:425-438.

15. Baloh RH, Schmidt RE, Pestronk A, Milbrandt J. Altered axonal mitochondrial transport in the pathogenesis of Charcot-Marie-Tooth disease from mitofusin 2 mutations. J Neurosci. 2007;27:422-430.

16. Hodgkinson VL, Dale JM, Garcia ML, et al. X-linked spinal muscular atrophy in mice caused by autonomous loss of ATP7A in the motor neuron. J Pathol. 2015;236:241-250.

17. Perez-Siles G, Grant A, Ellis M, et al. Characterizing the molecular phenotype of an: Atp7a T985I conditional knock in mouse model for X-linked distal hereditary motor neuropathy (dHMNX). Metallomics. 2016;8:981-992. 